\title{
Host immune response to Toxoplasma gondii and Ascaris lumbricoides in a highly endemic area: evidence of parasite co-immunomodulation properties influencing the outcome of both infections
}

\author{
Lílian MG Bahia-Oliveira ${ }^{1 /+}$, Juliana Azevedo da Silva ${ }^{1}$, Alba Lucinia Peixoto-Rangel', \\ Marcela Santana Bastos Boechat ${ }^{1}$, Annelise M Wilken Abreu Oliveira ${ }^{2}$, Cristiano L Massara ${ }^{3}$, \\ Ricardo Guerra Peixe ${ }^{1,2}$
}

\begin{abstract}
${ }^{1}$ Laboratório de Biologia do Reconhecer, Centro de Biociências e Biotecnologia, Universidade Estadual do Norte Fluminense, Av. Alberto Lamego 2000, 28013-620 Campos dos Goytacazes, RJ, Brasil ' Faculdade de Medicina de Campos, Campos dos Goytacazes, RJ, Brasil ${ }^{3}$ Laboratório de Helmintologia e Malacologia Médica, Instituto de Pesquisas René Rachou-Fiocruz, Belo Horizonte, MG, Brasil
\end{abstract}

Toxoplasmosis and ascaridiasis evoke polar Th-1 and Th-2 host immune responses, respectively. A study to investigate the specific cytokine profile production by in vitro cultures of peripheral blood mononuclear cells from individuals living under precarious sanitary conditions in a highly endemic area for the parasites Toxoplasma gondii and Ascaris lumbricoides was conducted. High levels of both IFN- $\gamma$ (Th-1) and IL-13 (Th-2) were observed in groups of co-infected individuals presenting toxoplasmic ocular lesions. Significantly lower IL-10 and TGF- $\beta$ levels were produced by co-infected individuals in comparison with groups of individuals not infected with A. lumbricoides and either positive or negative for $\mathrm{T}$. gondii living under good sanitary conditions (control groups). The possible influence of co-parasitism on the clinical presentation of ocular toxoplasmosis is discussed.

Key words: Toxoplasma gondii - Ascaris lumbricoides - uveitis - retinochoroiditis - environmental contamination - cytokines

Concurrent parasitic infections are common among individuals living under poor sanitary conditions in developing countries. It has been suggested that helminthic infections can influence health both negatively, i.e., by worsening protective responses against Mycobacterium tuberculosis in HIV-infected individuals (Bentwich et al. 1999), and positively, by improving the adaptive immune response against inflammatory diseases (Renz et al. 2006, Weinstock \& Elliot 2008).

Toxoplasmosis is generally asymptomatic in immunocompetent subjects. It may be acquired congenitally or at any time in life (Remington et al. 2006). Ocular toxoplasmosis is one of the main clinical manifestations in immunocompetent individuals and is considered a major cause of visual impairment (Holland 2003, 2004). The disease may be the result of recurring manifestations of congenital as well as acquired infections (Silveira et al. 1988, Holland et al. 1999). Many factors may influence the outcome of the disease, such as duration and frequency of exposure, route of infection, parasitic load, immunological factors and unidentified cofactors (McLeod et al. 1996, Garweg \& Candolfi 2009).

Type 1 cytokines are of fundamental importance in the immune response to Toxoplasma gondii in compe-

Financial support: CNPq (521234/96-4), PRONEX (2704), FAPERJ (E-26/171.162/96)

+ Corresponding author: 1ilian@uenf.br

Received 27 November 2008

Accepted 23 March 2009 tent hosts (Denkers \& Gazzinelli 1998). Ascaris lumbricoides infections, as with other gastrointestinal helminthic infections, are characterized by eosinophilia, mast cell hyperplasia and elevated levels of circulating IgE. In endemic areas for ascaridiasis, helminth infections were associated with a highly polarized type 2 cytokine response (Cooper et al. 2000).

The determination of the prevalence of toxoplasmic ocular lesions in areas highly endemic for T. gondii constitutes a challenge in practice. This prevalence may diverge in areas of high serum prevalence. In general, for epidemiological studies, subjective criteria for the classification of retinal lesions are adopted and lesions are often categorized in terms of the probability of being caused by T. gondii infection (Glasner et al. 1992).

The present study was undertaken to investigate the immune response parameters of ex-vivo phenotypic profiles, specific cellular proliferation and cytokine production by peripheral blood mononuclear cells (PBMCs) from individuals living in endemic areas for $T$. gondii and $A$. lumbricoides, and their possible relationship with the occurrence of diversified clinical sets of toxoplasmic retinochoroidal scars. The data obtained open new perspectives for the investigation of parasite-specific immunomodulators, with the potential to influence the outcome of toxoplasmosis and ascaridiasis.

\section{PATIENTS, MATERIAL AND METHODS}

Study population - A total of 88 individuals participated in this study. They were identified in a survey of the prevalence and risk factors for $T$. gondii infection in Campos dos Goytacazes, a city located in Northern of 
the state of Rio de Janeiro, with approximately 400,000 inhabitants and marked by economic and social divides (Bahia-Oliveira et al. 2003).

The following key variable definitions were adopted for the study population: (i) their sanitary conditions, (ii) the presence or absence of $T$. gondii and A. lumbricoides infections, and (iii) the presence or absence of retinal/ retinochoroidal scars. Sanitary conditions were defined as poor/precarious if neither residential sewage facilities nor municipally-treated water was available and if the monthly income of the household was less than US\$150. The $T$. gondii age-adjusted seroprevalence for this group was $84 \%$. Sanitary conditions were defined as good if the monthly income of the household was more than US\$500/ month and if treated water and sewage facilities were available. The $T$. gondii age-adjusted seroprevalence for this group was $23 \%$ (Bahia-Oliveira et al. 2003).

All of the 88 individuals were screened for the presence of helminth eggs (Hoffman et. al. 1934). Anti-helminth treatment (Mebendazol or Albenazol) was provided to all infected individuals. All the individuals living under precarious sanitary conditions (PSC) tested positive for $A$. lumbricoides and, of these individuals, some who were $T$. gondii-infected exhibited retinal/retinochoroidal lesion scars.

Individuals living in the same geographical area under good sanitary conditions (GSC), all of whom were negative for $A$. lumbricoides, comprised the control groups for this study. The control group individuals tested either negative or positive for toxoplasmosis; however, none of the positive ones presented retinal disorders. All groups were age and sex-matched and none were under drug therapy. None of the persons included had active inflammatory retinal lesions. No individual presented with clinical signs of malnutrition (WHO 1995).

The study protocol complied with the Helsinki Declaration and was approved by the Ethical Committee of Fundação Oswaldo Cruz-Fiocruz, Brazil, and the National Ethical Committee (CONEP 013/2007). Written informed consent was obtained from patients according to the guidelines of the Ethical Committee of Fiocruz.

Classification of the retinal/retinochoroidal scars - All the individuals presenting lesions were T. gondii-seropositive. The posterior retinal/retinochoroidal uveitis consistent with healed lesions was classified as either class $\mathrm{A}, \mathrm{B}$ or $\mathrm{C}$ according to their morphologic aspects, observed with a $20 \mathrm{D}$ or $78 \mathrm{D}$ lens and an indirect ophthalmoscope (Bahia-Oliveira et al. 2001, Oréfice $\&$ Bahia-Oliveira 2005). The lesions were photographed (Topcon TRC- 50X Retinal Fundus or digital ZEISS VISUCAM LITE Fundus). On average, each patient was examined by at least three ophthalmologists and a consensus was reached prior to lesion classification. The retinal/retinochoroidal scar size and localization were not considered as criteria in this classification; however, class $\mathrm{C}$ lesions were generally smaller than classes $\mathrm{A}$ and B. Class A lesions present well-marked boundaries, usually surrounded by a pigmented halo and extensive destruction of the retina and choroid. Class B lesions are characterized by a surrounding hypopigmented halo and a smaller degree of tissue destruction in comparison to class A. Class $\mathrm{C}$ lesions are basically areas of retinal pigmentar epithelium hyperplasia or atrophy with a smaller degree of tissue destruction compared to class A and B lesions, both of which fulfil all the morphological criteria of probably being caused by $T$. gondii infection. Because of the low degree of retinal destruction, class $\mathrm{C}$ lesions can be morphologically considered as a class of lesions of uncertain etiology.

Individuals presenting ocular lesions were examined twice, at the time of the survey and at the time this study was conducted (12-18 months after the survey). No difference was observed in any aspect of the lesions during this period.

Antigens - Antigenic preparations from T. gondii soluble tachyzoites antigen (STAg) were obtained from fibroblast cultures prepared as described previously (Gazzinelli et al. 1991) and used at a final concentration of $2 \mu \mathrm{g} /$ $\mathrm{mL}$. A. lumbricoides adult worms obtained from human faeces were used to prepare (ASC) antigen, derived from the whole body of the worm. Briefly, for ASC preparation, live worms from human faeces were washed several times with saline mixed with an equal volume of boratebuffered saline (BBS) pH 8.0 and homogenized in a potter. After centrifugation at $12,000 \mathrm{~g}$ for $1 \mathrm{~h}$, the precipitate was resuspended in BBS and stirred overnight at $4{ }^{\circ} \mathrm{C}$. The suspension was centrifuged again for $2 \mathrm{~h}$ at $12,000 \mathrm{~g}$ and the supernatant was dialysed against phosphate buffered saline $(0.15 \mathrm{M}, \mathrm{pH} 7.2)$, filtered through $0.45 \mu \mathrm{m}$ pore membranes for sterilization and used at a final concentration of $70 \mu \mathrm{g} / \mathrm{mL}$ (Soares et al. 1987). Staphylococcal enterotoxin B, SEB (SIGMA, St. Louis, USA) was used at a $0.25 \mu \mathrm{g} / \mathrm{mL}$ final concentration.

Cell preparation and proliferation assay - PBMCs were isolated by Ficoll-diatriazoate density gradient centrifugation (LSM, Organon Teknica, Charleston, SC, USA), as described previously (Gazzinelli et al. 1983). The culture medium consisted of $90.4 \%$ RPMI 1640 , $1.6 \%$ L-glutamine, $3 \%$ of an antibiotic-antimycotic mixture stock $(10,000 \mathrm{U}$ penicillin, $5 \mu \mathrm{g}$ streptomycin, $25 \mu \mathrm{g}$ fungizone per $\mathrm{mL}, \mathrm{GIBCO}$ ) and $5 \%$ heat-inactivated normal human serum AB, Rh+ (Sigma). For cytokine analysis, supernatants were collected from cultures of 1 x $10^{6}$ cells per well in flat-bottom 24-well tissue plates. For the cell proliferation assay, $2.5 \times 10^{5}$ cells were cultured in flat-bottom 96 -well tissue plates. Both types of plate cultures were maintained at $37^{\circ} \mathrm{C}$ in an atmosphere of $5 \% \mathrm{CO} 2$ for six days.

Cells were exposed to $0.5 \mu \mathrm{Ci}$ of tritiated thymidine (methyl-3H thymidine, Amersham Life Science, Buckinghamshire, UK) per culture (specific activity $2.0 \mathrm{Ci} / \mathrm{mM}$ ) for the last $6 \mathrm{~h}$ of culture and processed for scintillation counting. The data were calculated as mean counts per minute (CPM) of triplicate cultures and the results were expressed as experimental CPM - control (unstimulated) $\mathrm{CPM}(\mathrm{CPM}=\mathrm{E}-\mathrm{C})$.

Flow cytometry analysis - Ex vivo PBMCs were analyzed by cellular phenotypic analysis using two-colour flow cytometry in a Coulter EPICS ${ }^{\circledR}$ ELITE flow cytometer (Miami, FL, USA) for the following cell-surface markers: CD2, CD3, Pan $\gamma \delta, C D 4, C D 8, C D 16$ and 
CD19. A minimum of 3,000 gated (lymphocytes) cells were acquired. Monoclonal antibodies were purchased from Coulter Clone ${ }^{\circledR}$ (Miami, FL, USA), except for PEconjugated antibodies against CD16 and Pan $\gamma \delta$ (Immunotech-Coulter, Miami, USA).

Cytokine ELISA - Cytokine measurements were carried out by sandwich ELISAs using supernatants collected on different days according to previous kinetics studies performed in our laboratory (unpublished observations). One culture well was tested for each day supernatants were collected. Antibodies and standard cytokines were purchased from Pharmingen (San Diego, CA, USA) or R \& D systems (Minneapolis, MN, USA). IL-13, TNF- $\alpha$ and TGF- $\beta$ were measured in supernatants collected on the first day of culture, where the lower sensitivity cut-offs were $3.1 \mathrm{pg} / \mathrm{mL}, 5 \mathrm{pg} / \mathrm{mL}$ and $9 \mathrm{pg} /$ $\mathrm{mL}$, respectively. IL-5 and IL-6 were measured in supernatants collected on the third day of culture, where the lower cut-offs of sensitivity were $31.2 \mathrm{pg} / \mathrm{mL}$ and $0.6 \mathrm{pg} /$ $\mathrm{mL}$, respectively. IFN- $\gamma$, IL-12 and IL-10 were measured in supernatant collected on day six of culture, where the lower cut-offs of sensitivity were $15 \mathrm{pg} / \mathrm{mL}, 7 \mathrm{pg} / \mathrm{mL}$ and $6.3 \mathrm{pg} / \mathrm{mL}$, respectively.

Avidity of anti-T. gondii $\operatorname{Ig} G$ and anti-T. gondii $\operatorname{Ig} M$ antibodies - The levels of $T$. gondii specific IgM and IgG avidity were measured in the sera of all anti-T. gondii IgG positive patients using the BioMerieux VIDAS Kit (BIOMERIUEX, Lyon, France) according to the manufacturer's recommendations.

Statistical analysis - Phenotypic analysis results, scintillation count values for proliferation assays, and cytokine measurements were log transformed before performing statistical analyses. Comparisons between the groups were made by Student $t$ test, and $p \leq 0.05$ was considered as statistically significant with a $95 \%$ confidence interval.

\section{RESULTS}

Individuals were clustered in seven groups (Table) according to three parameters: (i) sanitary conditions, whether from areas of PSC or GSC; (ii) serology for $T$. gondii; and (iii) the presence (class A, B or C) or absence of scars of retinal/retinochoroidal lesions. Representative fundoscopic pictures of class A, class B and class C retinal/retinochoroidal lesion scars are shown in Fig. 1.

T. gondii-positive sera samples were tested for the IgM and IgG avidity index. All of the values were either lower or higher, respectively, than the cut-off values considered for recent (less than 4 months) infections (data not shown), indicating that the individuals were chronically infected. With the exception of group 3 (with class A ocular lesions), all T. gondii-seropositive groups had significantly higher levels of cellular proliferation against $T$. gondii STAg in comparison with seronegative groups (Table). The levels of cellular proliferation induced by ASC antigens did not differ across the groups. Additionally, these levels were very low, sometimes similar to those of cultures without antigenic stimulation. Control groups living under GSC, which were negative for A. lumbricoides, presented similar levels of cellular proliferation as those observed in the infected group living under PSC when their PBMCs were stimulated with ASC antigens (Table).

The mean level of $\gamma \delta \mathrm{T}$ cells was higher in group 5 (patients with ocular lesions, class C) when compared with all the other groups, although the difference was not statistically significant. The levels of cells expressing CD2/CD16 (NK cells) was significantly higher in group 2 (patients living under PSC without ocular le-

TABLE

Origin and mean age of Toxoplasma gondii-seropositive (SP) and seronegative (SN) individuals presenting the ex vivo percentage of NK and $\gamma \delta$ T cells, peripheral blood mononuclear cell proliferation values of cultures stimulated with Toxoplasma gondii and Ascaris lumbricoides antigens

\begin{tabular}{|c|c|c|c|c|c|c|c|c|}
\hline \multirow[t]{2}{*}{ Groups } & \multirow{2}{*}{$\begin{array}{c}\text { Toxoplasmosis } \\
\text { serology/ocular } \\
\text { lesion }\end{array}$} & \multirow{2}{*}{$\begin{array}{l}\text { Study } \\
\text { group }\end{array}$} & \multirow[t]{2}{*}{$\mathrm{n}$} & \multirow{2}{*}{$\begin{array}{l}\text { Mean Age } \\
\text { (SE) }\end{array}$} & \multirow{2}{*}{$\begin{array}{c}\text { NK cells } \\
\%(\mathrm{SE})\end{array}$} & \multirow{2}{*}{$\begin{array}{c}\gamma \delta \mathrm{T} \text { cells } \\
\%(\mathrm{SE})\end{array}$} & \multicolumn{2}{|c|}{ Antigenic stimulation } \\
\hline & & & & & & & $\begin{array}{l}\text { STAg } \\
* \Delta \mathrm{cpm}\end{array}$ & $\begin{array}{c}\mathrm{ASC} \\
* \Delta \mathrm{cpm}\end{array}$ \\
\hline 1 & SN & $\mathrm{PSC}^{e}$ & 18 & $20(4.7)$ & $7.85(0.94)$ & $4.01(0.84)$ & 1297.3 & $4676.9^{a}$ \\
\hline 2 & $\mathrm{SP} / \mathrm{NL}^{a}$ & $\mathrm{PSC}^{e}$ & 29 & $22(4.9)$ & $15.05(1.53) \mathrm{j}$ & $5.89(1.19)$ & $17400.2^{g, i}$ & 3494.3 \\
\hline 3 & $\mathrm{SP} / \mathrm{A}^{b}$ & $\mathrm{PSC}^{e}$ & 5 & $34(7.5)$ & $4.96(1.97)$ & $2.38(0.51)$ & $6982.1^{h}$ & 1653.0 \\
\hline 4 & $\mathrm{SP} / \mathrm{B}^{c}$ & $\mathrm{PSC}^{e}$ & 9 & $40(6.9)$ & $7.36(1.16)$ & $2.09(0.44)$ & $9194.4^{g, h}$ & $3125.2^{a}$ \\
\hline 5 & $\mathrm{SP} / \mathrm{C}^{d}$ & $\mathrm{PSC}^{e}$ & 6 & $28(4.5)$ & $8.92(2.76)$ & $8.02(4.75)$ & $6291.7^{g, h}$ & 2789.7 \\
\hline 6 & SN & $\mathrm{GSC}^{f}$ & 10 & $30(3.0)$ & $3.00(0.95)$ & $3.37(1.31)$ & 2521.3 & $3847.9^{a}$ \\
\hline 7 & $\mathrm{SP} / \mathrm{NL}$ & $\mathrm{GSC}^{f}$ & 11 & $34(3.2)$ & $4.33(0.98)$ & $4.62(0.67)$ & $12772.1^{g}$ & 2389.4 \\
\hline
\end{tabular}

Subjects belonging to groups 1-5 presented infected with A. lumbricoides; subjects belonging to groups 6 and 7 were negative for $A$. lumbricoides. a: (NL) T. gondii-seropositive individuals without ocular lesions; $b$-d: the retinochoroidal scars lesions categorized respectively as class A, B, or C; $e$ : precarious sanitary conditions (PSC) areas, all of the patients living in this area are infected with $A$. lumbricoides; $f$ : good sanitary conditions areas $(\mathrm{GSC})$; $g$ : significant difference $(\mathrm{p}<0.05)$ when compared to non-stimulated cultures; $h$ : significant difference $(\mathrm{p}<0.05)$ when compared to SN groups; $i$ : significant difference $(\mathrm{p}<0.05)$ when compared to SN groups; $j$ : significant difference when compared to groups $1(\mathrm{p}<0.05), 3(\mathrm{p}<0.01), 6$ and $7(\mathrm{p}<0.001)$; STAg: soluble tachyzoites antigen. Asterisk: $\Delta \mathrm{cpm}$ refers to values of cellular proliferation stimulated with antigens discounted from the values of cellular proliferation of the respective control cultures (not stimulated with antigens). 


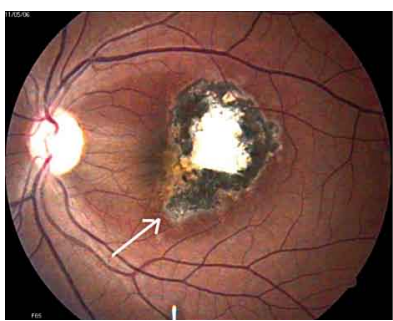

Type A lesion

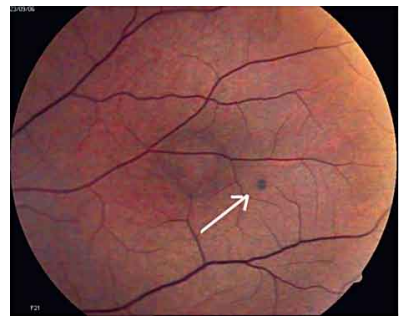

Type C lesion

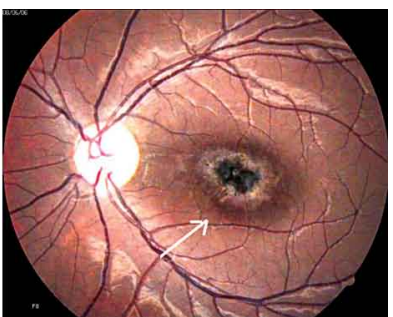

Type B lesion

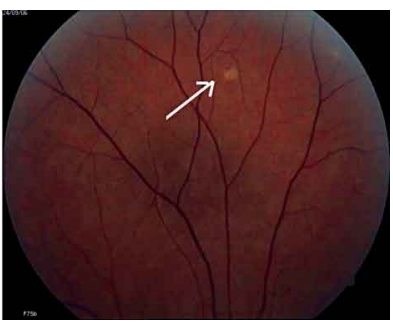

Type C lesion
Fig. 1: fundus photograph of representative retinochoroidal non active scar lesions type A, type B and type C. White arrows shows one lesion type $\mathrm{A}$, one lesion type $\mathrm{B}$ and two lesions type $\mathrm{C}$, respectively hyper and hypo pigmented.

sions) in comparison with all the other groups (Table). The CD4/CD8 ratio was not different between the studied groups (not shown).

The following cytokines were evaluated in PBMC supernatant cultures: IL-5, IL-6, IL-10, IL-12, IL-13, IFN- $\gamma$, TGF- $\beta$ and TNF- $\alpha$. Supernatants were collected on the day of the peak of each cytokine production as described in material and methods. Most of the cytokines had a peak-of-production day, except for IL-5, IL-6 and IL-10, which were continuously produced throughout the culture period (data not shown).
There were no significant differences between groups regarding the production of IL-5, IL-6 and IL-12 to SEB, parasitic antigens or media alone (data not shown). The levels of IL-5 in cultures stimulated with STAg and ASC antigens were generally lower than in control cultures not stimulated with antigens (data not shown).

The levels of IL-10 and TGF- $\beta$ detected in cultures stimulated with SEB, STAg and ASC are shown in Fig. $2 \mathrm{~A}, \mathrm{~B}$, respectively. No difference was observed between groups living under PSC, either with or without ocular lesions. However, patients living under GSC produced both cytokines at elevated levels $(p<0.05)$ in comparison with groups living under PSC in response to both antigens and in control cultures (not stimulated with antigens). Nevertheless, no differences were observed in cultures stimulated with SEB.

When stimulated with STAg, the mean levels of IL13 production in groups with ocular disease (class A, $\mathrm{B}$ and $\mathrm{C}$ lesions) were significantly higher than those observed for groups either negative or positive for toxoplasmosis and without lesions, as shown in Fig. 3A. The highest levels of IL-13 were secreted by PBMCs of patients belonging to the class $\mathrm{C}$ lesion group by stimulation with either STAg or ASC. All of the groups living under PSC secreted significantly higher levels of IL-13 under stimulation with ASC antigens in comparison with groups living under GSC (Fig. 3A); stimulation with SEB yielded no difference between the groups (data not shown). There was no significant difference between the levels of IL-13 secreted by PBMCs from toxo-seropositive and seronegative patients living under GSC (Fig. 3A) when stimulated with any antigenic preparation or SEB (data not shown).

The levels of TNF- $\alpha$ were low for the majority of the groups in response to both antigens (Fig. 3B). However, they were significantly higher in supernatants of cultures stimulated with STAg from subjects of group 2 ( $T$.
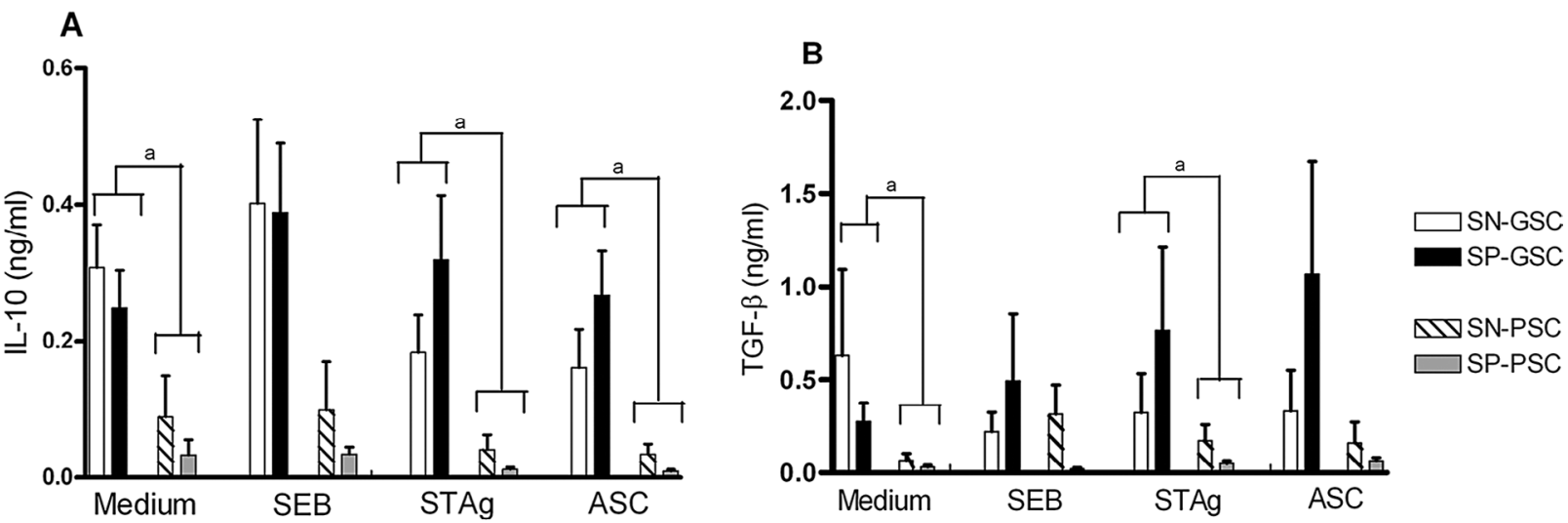

Fig. 2: in vitro cytokine production in supernatants of peripheral blood mononuclear cells (PBMC) cultures stimulated with SEB, soluble tachyzoites antigen (STAg) or ASC antigens. The mean levels of IL-10 and TGF- $\beta$ are respectively represented in panel A and B for patients grouped according to their Toxoplasma gondii serology, if positive (SP) or negative (SN), and according to their origin, if from GSC or PSC. The mean values represented are not discounted from the mean values of spontaneous production found for control cultures (without antigenic stimulation) that varied between $0.04 \mathrm{ng} / \mathrm{mL}$ and $0.55 \mathrm{ng} / \mathrm{mL}$ for IL-10 and $0.06 \mathrm{ng} / \mathrm{mL}$ and $0.63 \mathrm{ng} / \mathrm{mL}$ for TGF- $\beta$. The cytokine levels were statistically compared between groups of patients living under GSC and under PSC, independently of their $T$. gondii serology. Bars represent mean values plus standard medium error (+SEM). The statistical significance between mean values in the various groups was determined by One-way ANOVA followed by the Student's $t$ test. a: significant differences $(\mathrm{p}<0.05)$ between groups of individuals living in areas of precarious sanitary conditions (PSC) versus good sanitary conditions (GSC). 
gondii-seropositive patients without ocular lesions) than from $T$. gondii-seropositive patients with ocular lesions (groups 3, 4 and 5, $\mathrm{p}<0.05$ ).

The levels of IFN- $\gamma$ production are shown in Fig. $3 C$. These were markedly elevated $(p<0.05)$ in cultures stimulated with STAg from $T$. gondii-infected groups presenting ocular lesions and living under PSC in comparison with $T$. gondii-seropositive groups exhibiting no ocular lesions as well as seronegative groups living under PSC. The ASC antigens induced very low levels of IFN- $\gamma$ in PBMC cultures from all the groups studied. The highest levels of IFN- $\gamma$ were secreted by PBMCs from individuals presenting class $\mathrm{C}$ lesions by stimulation with STAg. There was no significant difference between the levels of IFN- $\gamma$ secreted by PBMCs from toxo-seropositive and seronegative individuals living under GSC (Fig. 3C).

\section{DISCUSSION}

Beyond parasite control, a better understanding of parasite immunomodulation can ultimately contribute to the development of medical applications. Many ex- amples illustrate this potential of parasites' molecules or extracts employed in experimental models. For example, the filarial nematode secreted product ES-62 has been shown to have both prophylactic and therapeutic efficacy in a murine model of arthritis (Harnett et al. 2004). Recently, anti-inflammatory ES-62 activity was found in synovial tissues from patients with rheumatoid arthritis (Harnett et al. 2008).

T. gondii and A. lumbricoides are both parasites that infect hosts orally; however, they elicit polar type I or type II host responses, respectively. Since both parasites are endemic in tropical areas, it is likely that co-infections with these organisms have been common throughout human evolution. If this is the case, then the host immune response mounted against both parasites may have adapted to permit such co-parasitism.

The epidemiological features of toxoplasmosis and ascaridiasis in the studied area must be considered in order to better understand the possible influence of each parasite on the outcome of infection by the other. Drinking water has been determined as the main risk factor for T. gondii infection for individuals living under PSC.
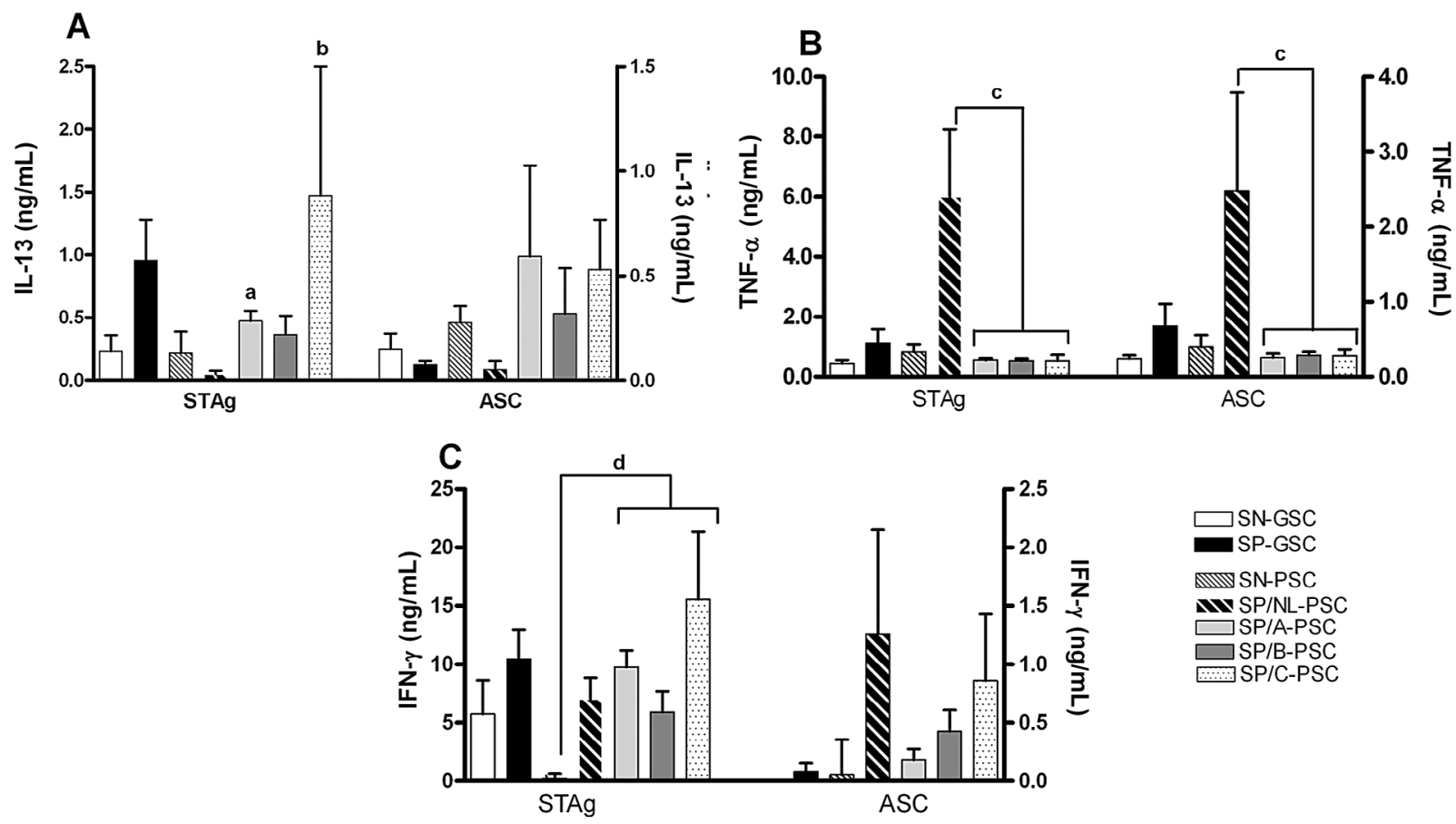

Fig. 3: in vitro cytokine production in supernatants of peripheral blood mononuclear cells (PBMC) cultures stimulated with soluble tachyzoites antigen (STAg) or ASC antigens. The mean levels of IL-13, TNF- $\alpha$ and IFN- $\gamma$ are respectively represented in panel A, B and C for patients grouped according to their Toxoplasma gondii serology, if negative (SN) or positive (SP), and according to their origin, if from good sanitary conditions (GSC) or from precarious sanitary conditions (PSC). The T. gondii seropositive patients, from PSC, are grouped according to the type of retinochoroidal scar A, B or C. The cytokine production mean values are represented in left $\mathrm{Y}$ axis for T. gondii in and right $\mathrm{Y}$ axis for Ascaris lumbricoides antigens. The mean values represented are not discounted from the mean values of spontaneous production found for control cultures (without antigenic stimulation) that varied between $0 \mathrm{ng} / \mathrm{mL}$ and $0.98 \mathrm{ng} / \mathrm{mL}$ for IL-13, $0.32 \mathrm{ng} / \mathrm{mL}$ and $3.29 \mathrm{ng} / \mathrm{mL}$ for TNF- $\alpha$ and $0.08 \mathrm{ng} / \mathrm{mL}$ and $1.57 \mathrm{ng} / \mathrm{mL}$ for IFN- $\gamma$. Cytokine levels were compared among individuals from GSC uninfected (SN/GSC) or infected (SP/GSC) with $T$. gondii and individuals from PSC grouped into five subgroups as following: patients presenting with class A (SP/A), class B (SP/B) and class $\mathrm{C}(\mathrm{SP} / \mathrm{C})$ ocular lesions, patients without ocular lesions (SP/NL) and T. gondii uninfected individuals (SN). Bars represent mean values plus standard medium error (+SEM). The statistical significance between mean values in the various groups was determined by One-way ANOVA followed by the Student's $t$ test. a: significant differences $(\mathrm{p}<0.05)$ between groups of patients with ocular lesion class A versus patients without ocular lesions; $b$ : significant differences $(p<0.001)$ between the group of patients with ocular lesion class $C$ versus patients without ocular lesions; c: significant differences $(\mathrm{p}<0.001)$ between group of patients with ocular lesions versus patients without ocular lesions; d: significant differences between group of patients with ocular lesions versus seronegative group of individuals $(p<0.001)$. 
T. gondii oocysts have been detected in consumable well water in the dwellings of individuals who took part in this study (unpublished observations). Considering that T. gondii oocysts are viable in water for long periods (Dubey 1998), we argue that, for the studied population, exposure to $T$. gondii antigens probably occurs very frequently and very early in life, according to the prevalence curve for the poor population involved the present study (Bahia-Oliveira et al. 2003). Exposure to A. lumbricoides is also likely to occur very frequently, since all the patients living under PSC were infected with the helminth and the prevalence of this infection is no lower than $90 \%$ in areas of dos Campos dos Goytacazes with poor sanitation (unpublished observations).

Late development of ocular toxoplasmic lesions can occur in cases of either congenital or acquired infection (Cooper et al. 2000). Because the present experimental setting did not address whether $T$. gondii infections were acquired or congenital, we will consider the profiles of immunologic parameters in light of the diversity of the clinical presentations of retinal/retinochoroidal scars found in T. gondii-seropositive patients co-infected with A. lumbricoides.

Generally, in endemic areas for A. lumbricoides, it has been shown that older individuals acquire resistance to the helminthic infection, which is associated with the cumulative capacity of older persons to produce anti-helminth IgE (Woolhouse et al. 1991). In the studied area, however, we did not observe this tendency. The in vitro immune response against $T$. gondii and $A$. lumbricoides antigens of co-infected individuals was marked by secretion of low levels of IL-10, IL-4, IL-5 and TGF- $\beta$ as well as by low levels of specific IgE against $A$. lumbricoides (data not shown). This aspect would favour the adaptation of the helminth to the host, since the specific protective immune response for that parasite is downregulated. The relatively low impact of toxoplasmic ocular lesions $(10-12 \%)$ is in contrast with the high serum prevalence of toxoplasmosis, which is $84 \%$ (adjusted for age) for persons of low socioeconomic income living in areas of precarious/poor sanitation in the present study. The $T$. gondii-specific immune response of patients presenting ocular lesions is marked by secretion of high levels of IFN- $\gamma$ and IL-13. Both cytokines might concomitantly and respectively limit the parasite's growth and control the inflammatory response within the eye, which would result in a better adaptation of $T$. gondii to the host. The higher levels of IL-13 secreted by PBMCs from co-infected individuals in comparison with the levels of this cytokine secreted by those infected only with $T$. gondii corroborate the idea that $A$. lumbricoides infection would favour the adaptation of $T$. gondii to the host, since the impact of eye infection would be lower (producing a less aggressive inflammatory response within the eye) and improve the chances of parasitism success. These data are in agreement with reports demonstrating that, in rats with endotoxin-induced uveitis, the systemic or intraocular injection of IL-13 significantly inhibited the production of pro-inflammatory cytokines and resulted in less intense ocular inflammation without down-regulating the levels of local IFN- $\gamma$ (Lemaitre et al. 2001). IL-13 has been considered an important cytokine for resistance to experimental intestinal nematode infections (Bancroft et al. 1998). However, the coinfected individuals did not secrete high levels of IL-13 under A. lumbricoides antigenic stimulation and its role in the helminthic infections for the studied population should be further investigated.

Aspects of the immune response of the studied individuals suggest that co-parasitism prompts the immune system to mount specific responses that differ from those observed in single-parasite infections. Considering the epidemiological and immunological aspects related to the studied area, we propose that (i) the coevolution might have resulted in a better adaptation for both parasites to their hosts and (ii) this adaptation was driven by products of parasites being able to modulate the host immune response.

Stoicov et al. (2004) demonstrated that $T$. gondii infection in a host infected with Helicobacter felis alters the natural outcome of $T$. gondii infection, allowing uncontrolled tachyzoite replication and severe organ damage and demonstrating the profound interactions of the immune response to unrelated organisms. Recently, Wagner et al. (2008), using a mouse model of birch pollen allergy, investigated whether infection with $T$. gondii influences allergic immune responses to birch pollen. It was demonstrated that $T$. gondii exhibits strong immunomodulating properties that lead to the prevention of Th-2 allergic immune responses. Curiously - and in contrast to our data - the infection was associated with enhanced TGF- $\beta$, IL-10 and Foxp3 mRNA, probably involved in suppression of the allergic immune response.

Anecdotal reports by local ophthalmologists support the view that recurrent $T$. gondii lesion activation rarely occurs in the studied area. Furthermore, no episode of lesion reactivation was reported during the 12-18 months when this study was conducted. If helminthic infections can improve the adaptive immune response against inflammatory diseases, then the host immune response to A. lumbricoides, which led to high levels of IL-13 production, may have benefited patients since it could potentially down-modulate pro-inflammatory cytokines without down-modulating IFN- $\gamma$ secretion, a cytokine required for $T$. gondii elimination (Suzuki et al. 1988).

Higher levels of IFN- $\gamma$, as well as IL-13, were observed in patients presenting class $\mathrm{C}$ lesions, which are characterized by less severe retinal damage, than in patients with class A and class B lesions. The high levels of IL-13 regulating the inflammatory process in the presence of high levels of IFN- $\gamma$ controlling $T$. gondii replication could, in principle, promote parasite growth control with minor tissue damage.

Because IFN- $\gamma$ and IL-13 are Th-1 and Th- 2 cytokines, respectively, it was expected that their levels would correlate inversely. Surprisingly, as estimated by Spearman's correlation, the secretion of these cytokines followed a positive correlation pattern instead, which, however, was not statistically significant (data not shown). We speculate that the positive correlation observed between IFN- $\gamma$ and IL-13 indicates that the cytokines do not inhibit the secretion of each other; rather, they might 
share a synergistic relationship. Evidence of synergism between IFN- $\gamma$ and IL-13 in the eye has been described in the experimental model of endotoxin-induced uveitis in rats where it was shown that IL-13 promoted a decreased expression of TNF- $\alpha$, IL-1 $\beta$, IL- 6 , monocyte chemoattractant protein - MCP-1 - and macrophage inflammatory protein - MIP-2 - as measured by mRNA levels in the iris-ciliary body and the retina while IFN- $\gamma$ was up-regulated in the iris-ciliary body (Lemaitre et al. 2001). That study thereby showed that the role of IL-13 in protecting against the damage of a strong inflammatory response in the eye.

Supporting this hypothesis are the data published by Yamamoto et al. (2000), showing that in the South of Brazil (living in the city of Erechim), where toxoplasmosis is highly prevalent and lesion reactivation is often observed (Silveira et al. 2001), the levels of IL-4 and IFN- $\gamma$ secretion were low in PBMC cultures from patients with eye lesions (Yamamoto et al. 2000). These differences might reflect variations in the clinical presentation of ocular diseases in both endemic areas of Brazil. Although toxoplasmosis is equally and highly prevalent in both areas $(\sim 80 \%)$, the prevalence of retinochoroiditis in Campos dos Goytacazes is much lower $(\sim 12 \%)$ than in Erechim $(\sim 25 \%)$. High genetic diversity of $T$. gondii isolates from Brazil has been described (Dubey et al. 2008) and the allelic diversity of parasite proteins, important in interactions with host cells, might partly explain the differences in the profile of host immune response as demonstrated by Saeij et al. (2007), who described ROP 16 interfering with STAT 3 and STAT 6 host transcription factors.

We did not quantify the parasitic helminthic burden in the present study and we therefore cannot infer its effect on the clinical presentation of the ocular lesions. Furthermore, because we have not evaluated the presence of other intestinal parasites, we cannot rule out the role of the response against other organisms that could elicit components of Th-2 or Th-1 immune responses.

The low degree of retinal destruction in group 3 (individuals with class $\mathrm{C}$ lesions) was also associated with higher levels of circulating $\gamma \delta$ T-cells compared to all the other groups studied. Elevated levels of $\gamma \delta$ T-cells have been associated with acute $T$. gondii infection (De Paoli et al. 1992). These results led us to investigate the levels of IgM and IgG avidity in their sera to estimate the duration of infection. Despite the cellular evidences of recent infections, the IgM and IgG avidity data did not confirm recent infection of less than four months. Therefore, it is not possible to appraise with much certainty whether class $\mathrm{C}$ lesions are more recently acquired lesions that will evolve to class A or B destructive lesions or whether they are an entity of lesions that are not normally registered, because they do not produce important symptoms. However, during the period of 12-18 months of this study, the registered $\mathrm{C}$ lesions did not evolve to class $\mathrm{A}$ or B, suggesting that they may constitute a class of toxoplasmic lesions that are not commonly detected, probably because of their low clinical impact or lack thereof, or because their evolution to class A or B is a rare and/ or slow event.
The significance of the high levels of TNF- $\alpha$ secreted by PBMCs from individuals with antibodies to $T$. gondii living under PSC and presenting no retinal scar tissue, as well as the presence of elevated levels of NK cells, remains to be further investigated in the context of protection against the development of toxoplasmic retinochoroiditis.

Although it is unclear whether the participating individuals had been infected congenitally or postnatally, we reason that at least some patients presenting class A lesions had been infected congenitally. Three parameters support this hypothesis: (i) the high prevalence of congenital toxoplasmosis in Campos dos Goytacazes (Bahia-Oliveira et al. 2001); (ii) the morphological aspects and the extent of tissue damage of class A lesions, since severe retinal/retinochoroidal damage is often observed in congenitally infected patients; and (iii) the fact that those individuals exhibited lower levels of PBMC proliferation under $T$. gondii stimulation in comparison with groups presenting no lesions or with other classes of lesions. In fact, the low capacity of PBMC proliferation in response to $T$. gondii in vitro stimulation has been associated with congenital infection, even in adults (Yamamoto et al. 2000). The observed low levels of PBMC proliferation in response to ASC stimulation is probably due to the inhibition of cellular proliferation that has been described for the murine model immunized with Ascaris suum antigens. High molecular weight components were responsible for its suppressive effect upon Th- 1 and Th2-dependent immune responses to unrelated antigens (Faquim-Mauro \& Macedo 1998).

Finally, the present study provides evidence for the importance of considering the natural conditions of antigenic exposure to commonly occurring parasites when investigating the role of immunologic parameters and adaptive immune responses to these parasites on disease outcomes.

The value of classifying toxoplasmic ocular lesions in terms of the degree of retinochoroidal destruction is highlighted by the data presented here. Follow-up of individuals presenting class $\mathrm{C}$ lesions, in this and other geographical areas, will be needed to improve our understanding and could potentially contribute to the development of immune therapy for ocular toxoplasmosis.

\section{ACKNOWLEDGEMENTS}

To Dr. David Addis (Centers for Disease Control and Prevention, Atlanta, Georgia, USA), Carlos Pavésio (Moorfields Hospital), Jack S. Remington (Palo Alto Medical Foundation, Stanford University) and Gary Holland (UCLA), for their support, to Juliana Salgado Viana, for their dedicated technical assistance, to the ophthalmologists Drs. Acácio Muralha, André Curi, Elisa Waked, Fernanda Porto, Fernando Oréfice, Gustavo Heringer, Lilia Muralha and Wesley Campos, for examining patients, and to Dr. Daíse Malheiros Meira, for her brilliant and original suggestion to propose a classification of the retinal/retinochoroidal scars in terms of the degree of retinochoroidal destruction.

\section{REFERENCES}

Bahia-Oliveira LMG, Jones J, Azevedo-Silva J, Alves CFC, Oréfice F, Addiss DG 2003. Highly endemic waterborne toxoplasmosis in North Rio de Janeiro Sate, Brazil. Emerg Infect Dis 9: 55-62. 
Bahia-Oliveira LMG, Wilken de Abreu AM, Azevedo-Silva J, Oréfice F 2001. Toxoplasmosis in Southeasthern Brazil in alarming situation of highly endemic acquired and congenital infection. In Recent trends in research on congenital toxoplasmosis, E Petersen, A Pollak, I Reiter-Owona (invited review), Intl J Parasitol 31: 115-144.

Bancroft AJ, McKenzie AN, Grencis RK 1998. A critical role for IL13 in resistance to intestinal nematode infection. J Immunol 160: 3453-3461.

Bentwich Z, Kalinkovich A, Weisman Z, Borkow G, Beyers N, Beyers AD 1999. Can eradication of helminthic infections change the face of AIDS and tuberculosis? Immunol Today 20: 485-487.

Cooper PJ, Chico ME, Sandoval C, Espinel I, Guevara A, Kennedy MW, Urban Jr JF, Griffin GE, Nutman TB 2000. Human infection with Ascaris lumbricoides is associated with a polarized cytokine response. J Infect Dis 182: 1207-1213.

Denkers EY, Gazzinelli RT 1998. Regulation and function of T-cellmediated immunity during Toxoplasma gondii infection. Clin Microbiol Rev 11: 569-588.

De Paoli P, Basaglia G, Gennari D, Crovatto M, Modolo ML, Santini G 1992. Phenotypic profile and functional characteristics of human gamma and delta $\mathrm{T}$ cells during acute toxoplasmosis. $J$ Clin Microbiol 30: 729-731.

Dubey JP 1998. Toxoplasma gondii oocyst survival under defined temperatures. J Parasitol 84: 862-865.

Dubey JP, Velmurugan GV, Chockalingam A, Pena HFJ, Nunes de Oliveira L, Leifer CA, Gennari SM, Bahia Oliveira LMG, Su C 2008. Genetic diversity of Toxoplasma gondii isolates from chickens from Brazil. Vet Parasitol 157: 299-305.

Faquim-Mauro EL, Macedo MS 1998. The immunosuppressive activity of Ascaris suum is due to high molecular weight components. Clin Exp Immunol 114: 245-251.

Garweg J, Candolfi E 2009. Immunopathology in ocular toxoplasmosis: facts and possible clues. Mem Inst Oswaldo Cruz 104: 211-220.

Gazzinelli RT, Hakim FT, Hieny S, Shearer GM, Sher A 1991. Synergistic role of CD4+ and CD8+ T lymphocytes in IFN-gamma production and protective immunity induced by an attenuated Toxoplasma gondii vaccine. J Immunol 146: 286-292.

Gazzinelli G, Katz N, Rocha RS, Colley DG 1983. Immune responses during human schistomiasis mansoni. VIII. Differential in vitro cellular responsiveness to adult worm and schistosomular tegumental preparations. Am J Trop Med Hyg 32: 326-333.

Glasner PD, Silveira C, Kruszon-Moran D, Martins MC, Burnier Junior M, Silveira S, Camargo ME, Nussenblatt RB, Kaslow RA, Belfort R Jr 1992. An unusually high prevalence of ocular toxoplasmosis in Southern Brazil. Am J Ophthalmol 114: 136-144.

Harnett MM, Kean DE, Boitelle A, McGuiness S, Thalhamer T, Steiger CN, Egan C, Al-Riyami L, Alcocer MJ, Houston KM, Gracie JA, McInnes IB , Harnett W 2008. The phosphorycholine moiety of the filarial nematode immunomodulator ES-62 is responsible for its anti-inflammatory action in arthritis. Ann Rheum Dis 67: 518-523.

Harnett W, McInnes IB, Harnett MM 2004. ES-62, a filarial nematode-derived immunomodulator with anti-inflammatory potential. Immunol Lett 94: 27-33.

Hoffman WA, Pons JA, Janer JL 1934. The sedimentation-concentration method in schistosomiasis mansoni. Puerto Rico J Public Health 9: 281-298.

Holland GN 2003. Ocular toxoplasmosis: a global reassessment. Part I: epidemiology and course of disease. Am J Ophthalmol 136: 973-988.
Holland GN 2004. Ocular toxoplasmosis: a global reassessment. Part II: disease manifestations and management. Am J Ophthalmol 137: 1-17.

Holland GN, Muccioli C, Silveira C, Weisz JM, Belfort R Jr, O'Connor GR 1999. Intraocular inflammatory reactions without focal necrotizing retinochoroiditis in patients with acquired systemic toxoplasmosis. Am J Ophthalmol 128: 413-420.

Lemaitre C, Thillaye-Goldenberg B, Naud MC, de Kozak Y 2001. The effects of intraocular injection of interleukin-13 on endotoxin- induced uveitis in rats. Invest Ophthalmol Vis Sci 42: 2022-2030.

McLeod R, Johnson J, Estes R, Mack D 1996. Immunogenetics in pathogenesis of and protection against toxoplasmosis. Curr Top Microbiol and Immunol 219: 95-112.

Oréfice F, Bahia-Oliveira L MG 2005. Toxoplasmose. In F Oréfice, Uveite clínica \& cirúrgica, Texto \& Atlas, Vol. II, 2 ed., Cultura Médica, Rio de Janeiro, p. 699-804.

Remington JS, McLeod R, Thulliez P, Desmonts G 2006. Toxoplasmosis. In J Remington, G Klein, C Wilson, C Baker, Infectious diseases of the fetus and newborn infant, 6th ed., WB Saunders, Philadelphia, p. 947-1091.

Renz H, Blumer N, Virna S, Sel S, Garn H 2006. The immunological basis of the hygiene hypothesis. Chem Immunol Allergy 91: 30-48.

Saeij JPJ, Coller S, Boyler JP, Jerome ME, White MW, Boothroy JC 2007. Toxoplasma co-opts host gene expression by injection of a polymorphic kinase homologue. Nature 445: 324-327.

Silveira C, Belfort R Jr, Burnier Jr M, Nussenblatt R 1988. Acquired toxoplasmic infection as the cause of toxoplasmic retinochoroiditis in families. Am J Ophthalmol 106: 362-364.

Silveira C, Belfort R Jr, Muccioli C, Abreu MT, Martins MC, Victora C, Nussenblatt RB, Holland GN 2001. A follow-up study of Toxoplasma gondii infection in Southern Brazil. Am J Ophthalmol 131: 351-354.

Soares MF, Macedo MS, Mota I 1987. Suppressive effect of an Ascaris suum extract on IgE and IgG antibody responses in mice. Braz J Med Biol Res 20: 203-211.

Stoicov C, Whary MK, Rogers AB, Lee FS, Klucevsek K, Li H, Cai X, Saffari R, Ge Z, Khan IA, Combe C, Luster A, Fox JG, Houghton JM 2004. Coinfection modulates inflammatory responses and clinical outcome of helicobacter felis and Toxoplasma gondii infections. J Immunol 173: 3329-3336.

Suzuki Y, Orellana MA, Schreiber RD, Remington JS 1988. Interferon-gamma: the major mediator of resistance against Toxoplasma gondii. Science 240: 516-518.

Wagner A, Förster-Waldl E, Garner-Spitzer E, Schabussova I, Kund M, Pollak A, Scheiner O, Joachim A, Wiedermann U 2008. Immunoregulation by Toxoplasma gondii infection prevents allergic immune responses in mice. Int J Parasit 39: 465-472.

Weinstock JV, Elliot DE 2009. Helminths and the IBD hygiene hypothesis. Inflamm Bowel Dis 15: 128-133.

WHO - World Health Organization 1995. Physical status: the use and interpretation of anthropometry. Report of a WHO Expert Committee. Technical Report Series 854, WHO, Geneva, 123 pp.

Woolhouse ME, Taylor P, Matanhire D, Chandiwana SK 1991. Acquired immunity and epidemiology of Schistosoma haematobium. Nature 351: 757-759.

Yamamoto JH, Vallochi AL, Silveira C, Filho JK, Nussenblatt RB, Cunha-Neto E, Gazzinelli RT, Belfort R Jr, Rizzo LV 2000. Discrimination between patients with acquired toxoplasmosis and congenital toxoplasmosis on the basis of the immune response to parasite antigens. J Infect Dis 181: 2018-2022. 